\title{
Posterior Spondylitis
}

- A 59-year-old patient

- Low back pain exacerbated by hyperextension

- Psoriasis
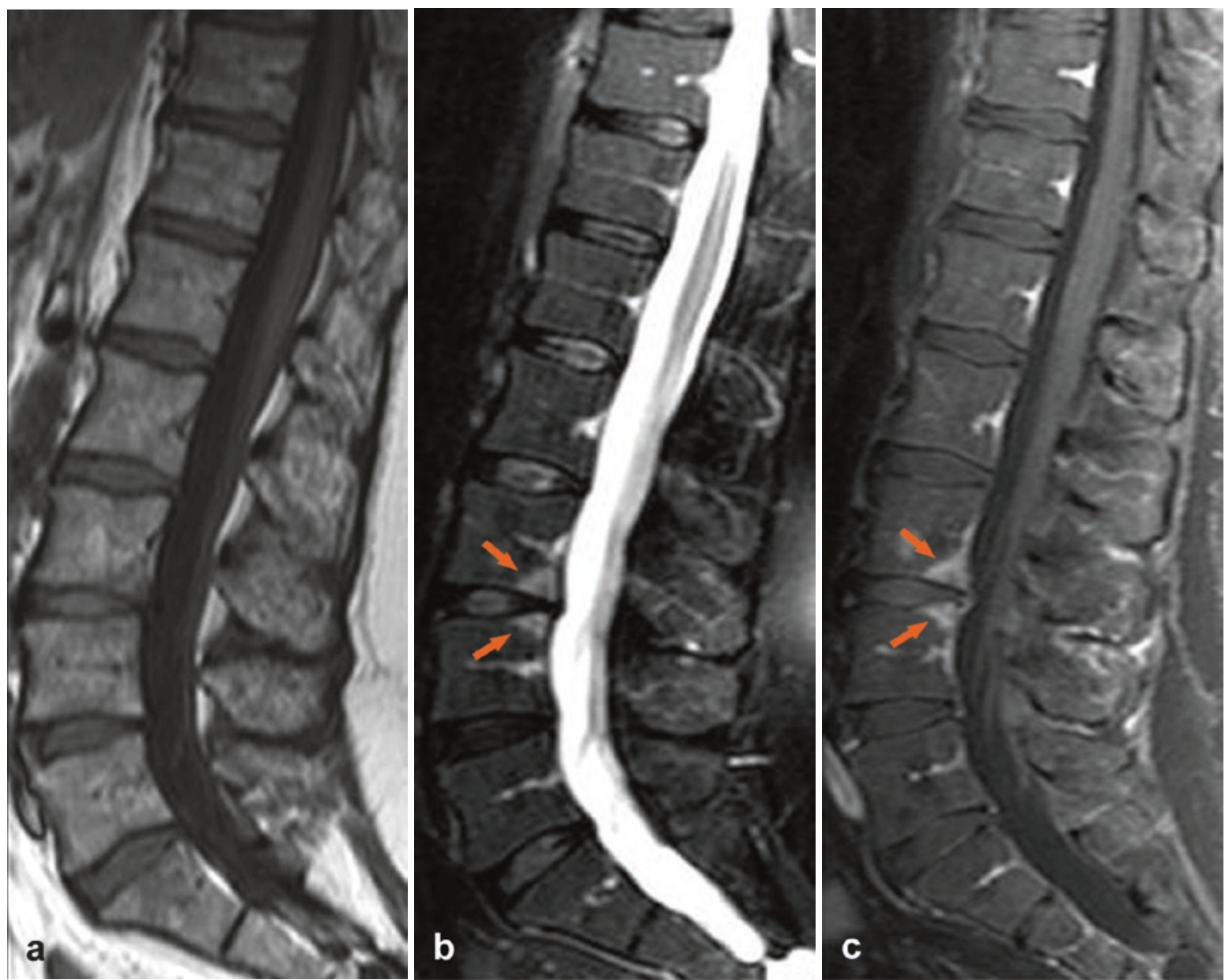

Fig. 1 Sagittal SE T1-weighted image (a), sagittal TSE T2-weighted image withfat saturation(b), sagittal SE T1-weighted image with fat saturation following the administration of contrast medium (c).
T2-hyperintensity and contrast enhancement in the posterior corners of the opposing vertebral bodies L3/L4 (b, c, arrows). Posterior spondylitis in active inflammatory phase 